\title{
Modelación no paramétrica de la contaminación promedio octohoraria del aire debida al Monóxido de Carbono y al Ozono Troposférico
}

ENVIROMENTAL ENGNEERING

\section{Nonparametric modeling of the eight-hour average air pollution due to Carbon Monoxide and Tropospheric Ozone}

\author{
Javier Olaya*§, Jhovana Reina*, Leidy L. Torres*, María C. Paz*, Luz A. Pereira* \\ * Escuela de Estadística, Universidad del Valle, Cali, Colombia \\ §javier.olaya@correounivalle.edu.co,jhoreina@gmail.com,leidytor2015@gmail.com,luz. \\ pereira@correounivalle.edu.co,karo.paz@gmail.com
}

(Recibido: 3 de Octubre de 2012 - Aceptado: 23 de Septiembre de 2013)

\begin{abstract}
Resumen
Se presenta la modelación del comportamiento típico de la concentración promedio octohoraria del monóxido de carbono $(\mathrm{CO})$ y ozono troposférico $\left(\mathrm{O}_{3}\right)$ a lo largo de un día, mediante la implementación de un modelo de regresión no paramétrica. Los datos utilizados provienen de las mediciones registradas por el Sistema de Vigilancia de la Calidad del Aire (SVCA) de la ciudad de Santiago de Cali, para los años 2003 y 2006. Los resultados obtenidos muestran que el comportamiento de la contaminación octohoraria por $\mathrm{O}_{3}$ supera el límite máximo permitido de la Norma Colombiana en las estaciones ubicadas en la zona sur de Cali, mientras que la contaminación octohoraria por $\mathrm{CO}$ se aproxima al límite máximo permitido en el centro de la ciudad, coincidente con la presencia de fuentes móviles. Las bandas de variabilidad asociadas a la modelación de la contaminación octohoraria del $\mathrm{CO}$, muestran indicios de que existen diferencias del comportamiento de este contaminante por tipo de día y zonas de vigilancia en los años de estudio; mientras que para el $\mathrm{O}_{3}$, el comportamiento difiere por tipo de día y zonas de vigilancia solo en el año 2006.
\end{abstract}

Palabras clave: bandas de variabilidad, contaminación del aire, estimador lineal local, regresión no paramétrica

\begin{abstract}
We model the typical behavior of the eight-hour average concentration of Tropospheric Ozone $\left(\mathrm{O}_{3}\right)$ and Carbon Monoxide (CO) throughout one day. Modeling is conducted using a nonparametric regression technique. Data come from the Cali's Air Quality Surveillance System (SVCA), and correspond to the years 2003 and 2006. Results show that in the Cali's South side, the $\mathrm{O}_{3}$ eight hour contamination levels exceed the Colombian standard limit value. Meanwhile, in Cali's downtown the $\mathrm{CO}$ eight-hour average contamination levels are very close to the Colombian standard limit. Variability bands associated with the modeling of the typical daily behavior of the eight-hour concentration of $\mathrm{CO}$, show evidence of differences between types of day and also between monitoring zones in all the years considered in the project. The same is observed for the $\mathrm{O}_{3}$ case, but only for data coming from year 2006.
\end{abstract}

Keywords: air pollution, local linear estimator, nonparametric regression, variability bands. 


\section{Introducción}

El control de las concentraciones de los contaminantes en la atmósfera es un tema de vital interés a nivel mundial. No sólo en los niveles que se presentan problemas graves para la salud humana, sino también cuando estas concentraciones no representan mayores inconvenientes para los seres humanos. Entre los contaminantes considerados como peligrosos en la atmosfera, se encuentra el Ozono Troposférico $\left(\mathrm{O}_{3}\right)$ y el Monóxido de Carbono (CO), (U.S. EPA, 1990). Varios estudios dan indicio de los efectos tóxicos en personas expuestas a altos niveles de estos contaminantes durante ciertos periodo de tiempos, lo cual trae como consecuencia graves efectos en la salud humana, como enfermedades cardiovasculares, respiratorias, etc., (U.S. EPA, 2000; 2006; Pantazopoulou et al.,1995; Poloniecki et al., 1997; Schwartz et al., 1999). Estos dos contaminantes tienen efectos nocivos importantes sobre la fauna, flora y sobre materiales de uso común, además hacen parte de los gases que causan el efecto invernadero que se presenta en la actualidad (U.S. EPA, 2000, 2006).

El Monóxido de Carbono (CO) se conoce comúnmente como un "Asesino Invisible" (CSPC, 2013), ya que sus propiedades químicas hacen de este un contaminante inodoro, incoloro e insípido. La tercera parte de las emisiones de Monóxido de Carbono se atribuye a diversos procesos naturales, mientras que los dos tercios restantes son el resultado de actividades humanas, como por ejemplo; la quema de materiales que contienen gas, gasolina, querosén, carbón, petróleo, madera (U.S. Department of Labor Occupational Safety and Health Administration, 2002), los carros detenidos con el motor encendido, las chimeneas, estufas, calentadores de agua, etc. son algunos ejemplos de procesos que dan origen al CO (U.S. EPA, 2000).

En la troposfera de la tierra, el Ozono se vuelve "malo" (U.S. EPA, 2003) al reaccionar químicamente en presencia de la luz solar con contaminantes como el Óxido de Nitrógeno $\left(\mathrm{NO}_{\mathrm{x}}\right)$ y Compuestos Orgánicos
Volátiles (VOC), los cuales son emitidos por automóviles, industrias, plantas generadoras de energía, refinerías, plantas químicas; entre otras fuentes, causando efectos perjudiciales sobre las personas que lo respiran (U.S. EPA, 2006).

La problemática de la contaminación atmosférica, no es ajena a las ciudades de Colombia, la población de éste país tiende a concentrarse cada vez más en las principales ciudades (IDEAM, 2007), entre las cuales se encuentra la ciudad de Santiago de Cali, municipio del Valle del Cauca ubicado al suroccidente de Colombia. Esta ciudad cuenta con un Sistema de Vigilancia de la Calidad del Aire (SVCA) que inició operaciones a partir del año 1999. Luego de haber dejado de funcionar durante cuatro años consecutivos, debido a la falta de mantenimiento, en el año 2003 reinició su funcionamiento, el cual volvió a culminar en el año 2006. La SVCA para el año 2010 arranca en funcionamiento en solo dos estaciones de vigilancia: Estación ERA (Zona Centro) y Estación Éxito de la Flora..

Una medida estándar (U.S. EPA, 2000; 2006) de los riesgos de la exposición prolongada al $\mathrm{CO}$ y $\mathrm{O}_{3}$, es el promedio móvil de 8 horas de las concentraciones horarias de cada contaminante, razón por la cual, este proyecto se concentra en modelar los promedios móviles de 8 horas de $\mathrm{CO}$ y $\mathrm{O}_{3}$ a lo largo de un día, en la ciudad de Santiago de Cali, durante los años 2003 y 2006; por año, zona de vigilancia y tipo de día. Los promedios móviles de 8 horas de los contaminantes $\mathrm{CO}$ y $\mathrm{O}_{3}$, fueron calculados a partir de los registros de promedios horarios suministrados por el Departamento Administrativo para la Gestión del Medio Ambiente (DAGMA), medidos en 8 estaciones de vigilancia de la ciudad de Cali.

El análisis de este proyecto se encuentra enmarcado dentro de la Norma Colombiana de Calidad del Aire (Artículo 4, Capitulo II, Resolución 601 DE 2006), la cual permite identificar zonas de vigilancia que sobrepasan los límites máximos permitidos por el $\mathrm{CO}$ y $\mathrm{O}_{3}$ para los años 2003 y 2006. Los contaminantes se analizan separadamente, siguiendo la Norma Colombiana de Calidad del Aire. Se espera que 
los resultados encontrados en este proyecto sean una herramienta fundamental para la toma de decisiones en cuanto a la gestión de políticas de calidad del aire.

La metodología aplicada en este trabajo para modelar el comportamiento de los contaminantes fue la Regresión no Paramétrica, a partir del Estimador Lineal Local, un suavizador basado en funciones tipo Kernel (Cleveland, 1979). Esta técnica estadística permite realizar estimaciones sin suponer una función a priori al comportamiento de los datos, en comparación a la regresión paramétrica, siendo una herramienta flexible para la construcción de modelos que no necesariamente cumplen con el supuesto de linealidad, (Bowman \& Azzalini, 1997; Eubank, 1999; Härdle, 1992).

En este artículo, se propone una adaptación del estimador de varianza utilizado para la construcción de bandas de variabilidad asociadas a la estimación global de los modelos estimados para cada contaminante, ya que teóricamente se ha tratado muy poco el problema de estimación de varianza cuando se tienen múltiples observaciones por punto de diseño.

\section{Metodología}

\subsection{Descripción de los datos}

Losdatosutilizados en esteestudiocorresponden a los promedios móviles de 8 horas (8-h) de las concentraciones horarias del Monóxido de Carbono (CO) y Ozono Troposférico $\left(\mathrm{O}_{3}\right)$, los cuales fueron calculados a partir de los registros de promedios horarios suministrados por el Departamento Administrativo de Gestión del Medio Ambiente (DAGMA), medidos en las siguientes estaciones de vigilancia: Centro Diagnóstico Automotor del Valle (CDAV), Base Aérea Marco Fidel Suárez (BA), Polideportivo el Diamante (PDD), CVC Pance, Escuela República Argentina (ERA), Universidad del Valle (UV) y estación Calle 15, para los años 2003 y 2006. Los registros de los niveles de concentración de los contaminantes son valores promediados cada hora; es decir, valores de concentración horaria (1-h) medidos en partes por millón (ppm) para $\mathrm{CO}$ y partes por billón (ppb) para $\mathrm{O}_{3}$.

Para efectos de análisis, el formato de la hora en la cual se capturó el registro de los valores de $\mathrm{CO}$ fue modificado a formato numérico, de tal manera que la hora 0 representa la primer hora del día y comprende el periodo de tiempo transcurrido entre las 0:00 a.m. y las 0:59 a.m.; y la hora 23 es la última hora del día que representa al periodo de tiempo transcurrido entre las 11:00 p.m. y las 11:59 p.m.

\subsection{Modelo de regresión no paramétrico: estimador lineal local}

Varios autores, Nadaraya \& Watson (1964), Cleveland (1979), Gasser \& Müller (1979), Priestley \& Chao (1972), han propuesto diversos estimadores o suavizadores no paramétricos que permiten describir el comportamiento característico de un conjunto de datos, sin suponer a priori una función de regresión conocida. Supóngase para un conjunto de observaciones $\left(x_{i}, y_{i}\right) ; i=1,2, \ldots, n$, la relación de regresión expresada por la siguiente forma:

$$
y_{i}=f\left(x_{i}\right)+\varepsilon_{i} ; i=1,2, \ldots, n
$$

donde $y_{i}$ representa la variable dependiente, $x_{i}$ los valores de la variable independiente o de diseño, $f\left(x_{i}\right)$ la función de regresión $\mathrm{f}$ desconocida evaluada en los puntos de diseño, y $\varepsilon_{i}$ una variable aleatoria denominada error. Un objetivo del análisis de regresión es obtener una aproximación razonable de la función desconocida $\mathrm{f}$, reduciendo el error de las observaciones y centrándose en la respuesta media de $\mathrm{y}$, condicionada a $\mathrm{x}$, lo que se expresa como: $f\left(x_{i}\right)=E\left(Y \mid X=x_{i}\right)$ con $E\left(\varepsilon_{i}\right)=0 \mathrm{y}$ $\operatorname{Var}\left(\varepsilon_{i}\right)=\sigma^{2}$ para todo i.

Los estimadores de regresión no paramétrica tienen como fin estimar la forma de la función $\mathrm{f}$ a través de técnicas de suavización (Kernel, K- Vecinos más cercanos, Spline, etc.), sin necesidad de especificar a priori su estructura. La filosofía de estos métodos se basa en la idea de dejar que los datos hablen por sí mismos, Olaya (2005). 
Eubank (1999), especifica que en regresión no paramétrica, se debe asumir que $\mathrm{f}$ es suave; es decir, que si se desea estimar la función $\mathrm{f}$ en un punto $\mathrm{x}$, se espera que las observaciones $y_{i}$ asociadas a los $x_{i}$ cercanos a $x$, posean información de $\mathrm{f}$ en $\mathrm{x}$, lo cual indica que es posible promediar de alguna forma las respuestas $\mathrm{y}_{\mathrm{i}}$ más cercanas al punto donde se estime $\mathrm{f}(\mathrm{x})$. Los estimadores más comunes suelen tener una estructura lineal en la variable dependiente, es decir, se expresa como una función lineal de la variable respuesta.

La técnica de suavización conocida como Kernel, hace uso de una función que asigna pesos a las observaciones lejanas o cercanas al punto de interés. La función que asigna pesos en esta técnica de suavización está determinada por una función Kernel y por un parámetro de suavización, Härdle (1992).

Las funciones Kernel en general, son funciones de densidad de probabilidad simétrica, cuyo pico ocurre en cero, Eubank (1999). La función de pesos decrece a cero a medida que se aleja del punto de estimación $x$ y está definida como:

$$
W\left(x, x_{i} ; \lambda\right)=\frac{1}{\lambda} K\left(\frac{x-x_{i}}{\lambda}\right)=\frac{1}{\lambda} K(u)
$$

Donde $K(u)$ es una función kernel y $u=\frac{x-x_{i}}{\lambda}$. Las funciones Kernel utilizan ciertas propiedades ó condiciones de momentos en el cual se define el orden del Kernel. La función de pesos depende del parámetro de suavización seleccionado, el cual permite otorgar mayor peso a los puntos cercanos al punto donde se va a suavizar (punto de interés) y un peso bajo a los puntos que están alejados del mismo.

Los estimadores Kernel se pueden obtener como solución de un problema de mínimos cuadrados ponderados en polinomios de diferentes grados, Cleveland (1979). Se considera que los polinomios de grados mayores a uno, aumentan la flexibilidad de la regresión, reduciendo el sesgo pero introduciendo mayor variabilidad.

En el caso de que el polinomio sea de grado $p$ $=0 \min _{\alpha} \sum_{i=1}^{n}\left\{y_{i}-\alpha\right\}^{2} w\left(x-x_{i} ; \lambda\right)$ se obtiene el Estimador Promedio Local o Estimador de Nadaraya-Watson, para $p=1$ se obtiene el Estimador Lineal local. En ambos casos se toma como estimación en $\mathrm{x}$ el valor de $\alpha$ en el cual se intercepta la recta ajustada y la recta $\mathrm{X}=\mathrm{x}$, Bowman \& Azzalini (1997).

El Estimador Lineal Local tiene ventajas sobre el Estimador Promedio Local. Una de ellas es debida a que cuando $\lambda$ aumenta, los pesos dados por la función Kernel se acercan más y la curva estimada se aproxima a una línea de regresión por mínimos cuadrados. En cambio el estimador promedio local tiende a ser una línea paralela al eje de las $\mathrm{x}$ con intercepto en $\bar{y}$, Cleveland (1979).

A partir de lo anterior, se tiene que los modelos ó curvas típicas estimadas para el comportamiento octohorario del $\mathrm{CO} \mathrm{y}_{3}$ se construyen a partir del siguiente modelo:

$$
y_{i}=\mu\left(x_{i}\right)+\varepsilon_{i}
$$

donde $y_{i}$ es el nivel de concentración octohoraria del $\mathrm{CO}$ (ppm) ó $\mathrm{O}_{3}$ (ppb) según sea el caso, $x_{i}$ es la hora del día $(0,1,2, \ldots, 23)$ y $\mu\left(x_{i}\right)$ es la estimación de la curva típica diaria de las concentraciones octohorarias de $\mathrm{CO}$ ó $\mathrm{O}_{3}$, según sea el caso. La modelación de los niveles de concentración 8-h de $\mathrm{CO}$ y $\mathrm{O}_{3}$ se estimó mediante la técnica de suavización Kernel. El suavizador utilizado a partir de esta técnica es el Estimador Lineal Local propuesto por Cleveland (1979). La función Kernel Gaussiana fue utilizada para la asignación de pesos a los datos. El parámetro de suavización o ancho de banda se estimó mediante una minimización de una medida global del error llamada Error Cuadrático Integrado Medio (MISE), Bowman \& Azzalini (1997), Härdle (1992). El método utilizado para la estimación de esta medida fue a través del criterio de la Validación Cruzada, Bowman \& Azzalini (1997).

En el análisis de contaminación atmosférica, es importante tener en cuenta medidas que controlen los niveles de contaminación, lo cual se puede lograrse a partir de "límites de alerta", mediante la estimación de valores estadísticos. Es por esto que en este artículo, se propone la 
utilización de bandas de variabilidad, Bowman \& Azzalini (1997) asociadas a la estimación global de los modelos estimados. Por lo tanto, la estimación de varianza juega un papel importante en este estudio, ya que esta permite la estimación de límites de detección, Hall \& Carrol (1989) por medio de las bandas de variabilidad asociadas a cada curva o modelo de regresión estimado.

Debido a lo anterior, se sugiere una adaptación del estimador de varianza para el caso de múltiples respuestas, propuesto por Seifert et al. (1993), teniendo en cuenta el estimador de Gasser et al. (1986) de forma secuencial en el tiempo, ya que poco se ha estudiado acerca del problema de estimación de varianza cuando se poseen múltiples observaciones por punto de diseño, Gasser et al., (1993).

Las bandas de variabilidad se construyen a partir del error estándar de la estimación de la curva Bowman \& Azzalini (1997). El error estándar de la curva de regresión está dado por:

$$
\operatorname{se}\{\hat{\mu}(x)\}=\sqrt{\operatorname{var}\{\hat{\mu}(x)\}}=\sqrt{\operatorname{diag}\left(S \times S^{T}\right)} \hat{\sigma}^{2}
$$

donde $S$ es la matriz de suavizamiento para el estimador lineal local, $\hat{\mu}(x)$ es la estimación de la curva de regresión no paramétrica y $\hat{\sigma}^{2}$ es la estimación de varianza de los errores.

\section{Resultados y discusión}

La metodología utilizada en la estimación de las curvas que representan la concentración 8-h para $\mathrm{CO} \mathrm{y}_{3}$, según el tipo de día fue la suavización kernel. Todas las estimaciones se realizaron con el paquete estadístico R (R Core Team, 2012), bajo el módulo sm.regression de la librería sm desarrollada por Bowman y Azzalini (2010).

Dado que el monóxido de carbono y los contaminantes precursores del ozono, son producidos en mayoría por actividades humanas, se analizó el comportamiento de estas actividades a partir del día de la semana. Mediante el análisis exploratorio de datos, se encontró que las concentraciones octohorarias de $\mathrm{CO}$ y $_{3}$ presentaron un comportamiento característico por el tipo de día. Por esta razón, para el CO se estimaron curvas típicas de las concentraciones 8-h por la siguiente clasificación: Lunes, Martes-Viernes y Fin de Semana, por año y estación de vigilancia. Para el $\mathrm{O}_{3}$, el método de clasificación fue: LunesViernes, Sábados y Domingos-Festivos.

A partir de los registros de los niveles de concentración promedio horarios de $\mathrm{CO}$ y $\mathrm{O}_{3}$ suministrados por el SVCA del DAGMA y de acuerdo al Protocolo de Monitoreo y Seguimiento de Calidad del Aire, el cual es elaborado por el Instituto de Hidrología, Meteorología y Estudios Ambientales (IDEAM, 2005), se procedió al cálculo de las concentraciones 8-h, estas últimas se refieren al valor que toma la concentración de un contaminante en una hora determinada, cuando se calcula a partir de un promedio móvil de ocho horas.

El cálculo de la concentración 8-h para una hora determinada corresponde al promedio aritmético de ocho concentraciones horarias, entre las cuales están la concentración horaria para la hora de cálculo y las siete concentraciones horarias anteriores a esa hora. La primera hora de cálculo (hora 0 ) para un día cualquiera será el período comprendido entre la hora 17 del día anterior hasta la hora 0 de dicho día, la última hora de cálculo (hora 23) para un día cualquiera será el período a partir de la hora 16 hasta la hora 23 de dicho día.

Según la Figura 1, en todas las estaciones de vigilancia el comportamiento de la concentración 8-h de $\mathrm{O}_{3}$, se releja en una curva que inicia con valores bajos de concentración y que decrecen hasta las 9 a.m., hora en la cual inicia un incremento que alcanza una concentración máxima aproximadamente a las 5:00 p.m. y después empieza de nuevo un descenso del nivel de concentración hasta finalizar el día.

La Figura 1 ilustra el comportamiento del $\mathrm{O}_{3} 8$-h en el año 2003, según el tipo de día. Se observa que en todas las estaciones de vigilancia las concentraciones estimadas poseen valores muy cercanos al máximo permitido por la norma de 

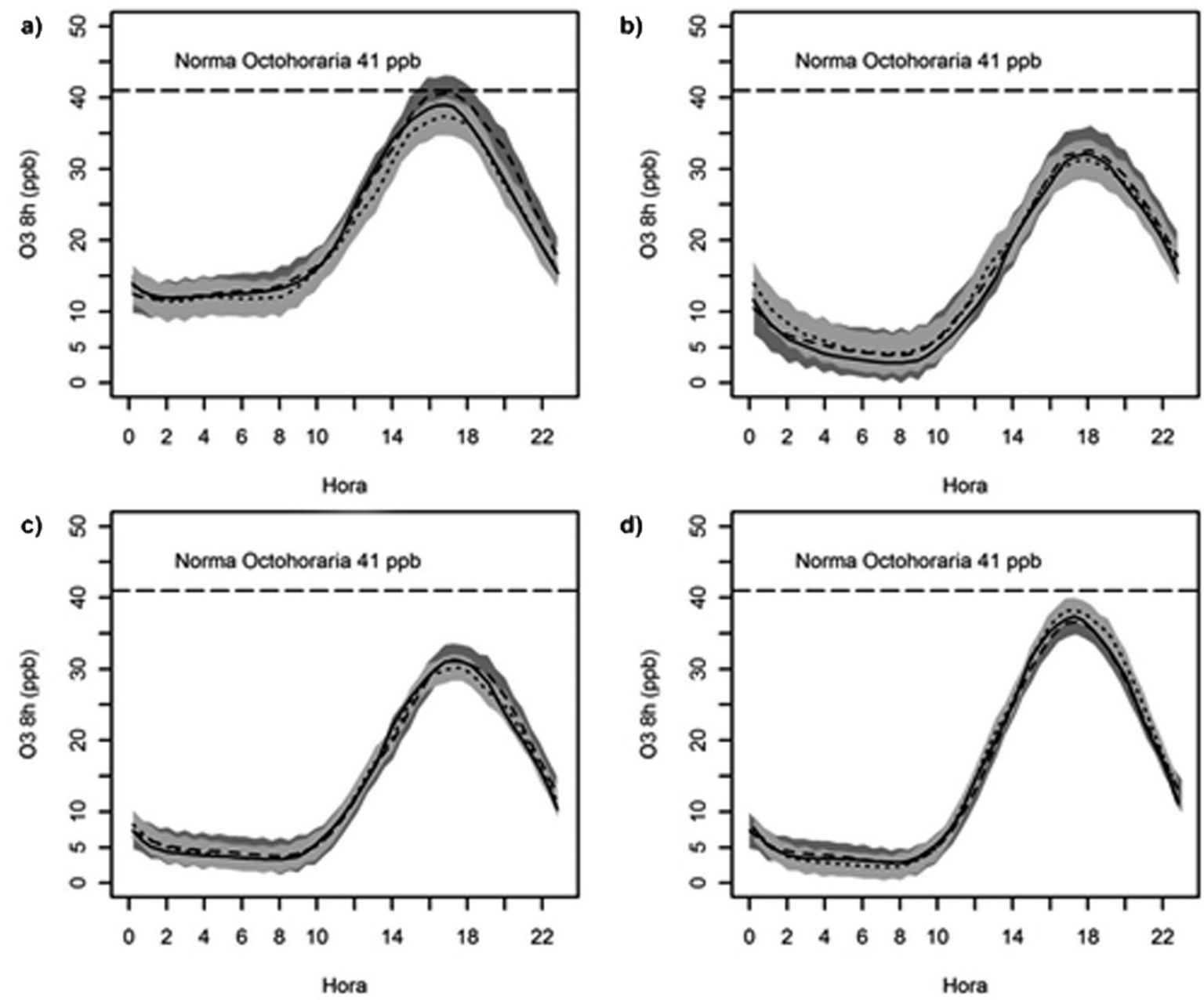

Figura 1. Curvas típicas y bandas de variabilidad de los niveles de concentración octohoraria de O3 en la estación Centro de Diagnóstico Automotor del Valle (a), estación CVC (b), estación Polideportivo el Diamante (c) y estación Univalle (d), para los días Lunes-Viernes (-), Sábados (--) y Domingos-Festivos (...), año 2003.

calidad del aire en Colombia. En particular, la estación CDAV presenta concentraciones de $\mathrm{O}_{3} 8$-h que sobrepasan el límite máximo permisible $(41 \mathrm{ppb})$ en horas de la tarde para los días sábados.

La Figura 2, ilustra el comportamiento del $\mathrm{O}_{3}$ en el año 2006, según el tipo de día. Se observa que en la estación CDAV se presentaron las menores concentraciones de $\mathrm{O}_{3}$ en el año 2006, según el tipo de día 8-h en comparación al comportamiento observado en el año 2003. Los días sábados presentan concentraciones que sobrepasan el máximo permitido en las estaciones ubicadas en el sur de la ciudad (Univalle y CVC).

La Figura 3 muestra el comportamiento de la contaminación de CO 8-h en el año 2003, según el tipo de día. En general, se observa que el tipo de día en el cual se presentaron mayores niveles de contaminación 8-h fue en los días martes-viernes. Para los días fines de semana, se muestra el mismo comportamiento que en los días ordinarios, solo que a menor escala, indicando una posible disminución de contaminación durante estos días.

Cabe destacar que la zona centro (Calle 15), ha sido la zona más afectada por la contaminación 8-h de CO, principalmente en los días MartesViernes, debido muy posiblemente al alto tráfico de motores vehiculares en la zona centro de la ciudad. La curva asociada a la estación Calle 15 para todos los tipos de días, muestra un pico máximo de contaminación de CO 8-h alrededor de las 4:00 p.m. En ninguno de los casos, las concentraciones 8 -h de este 

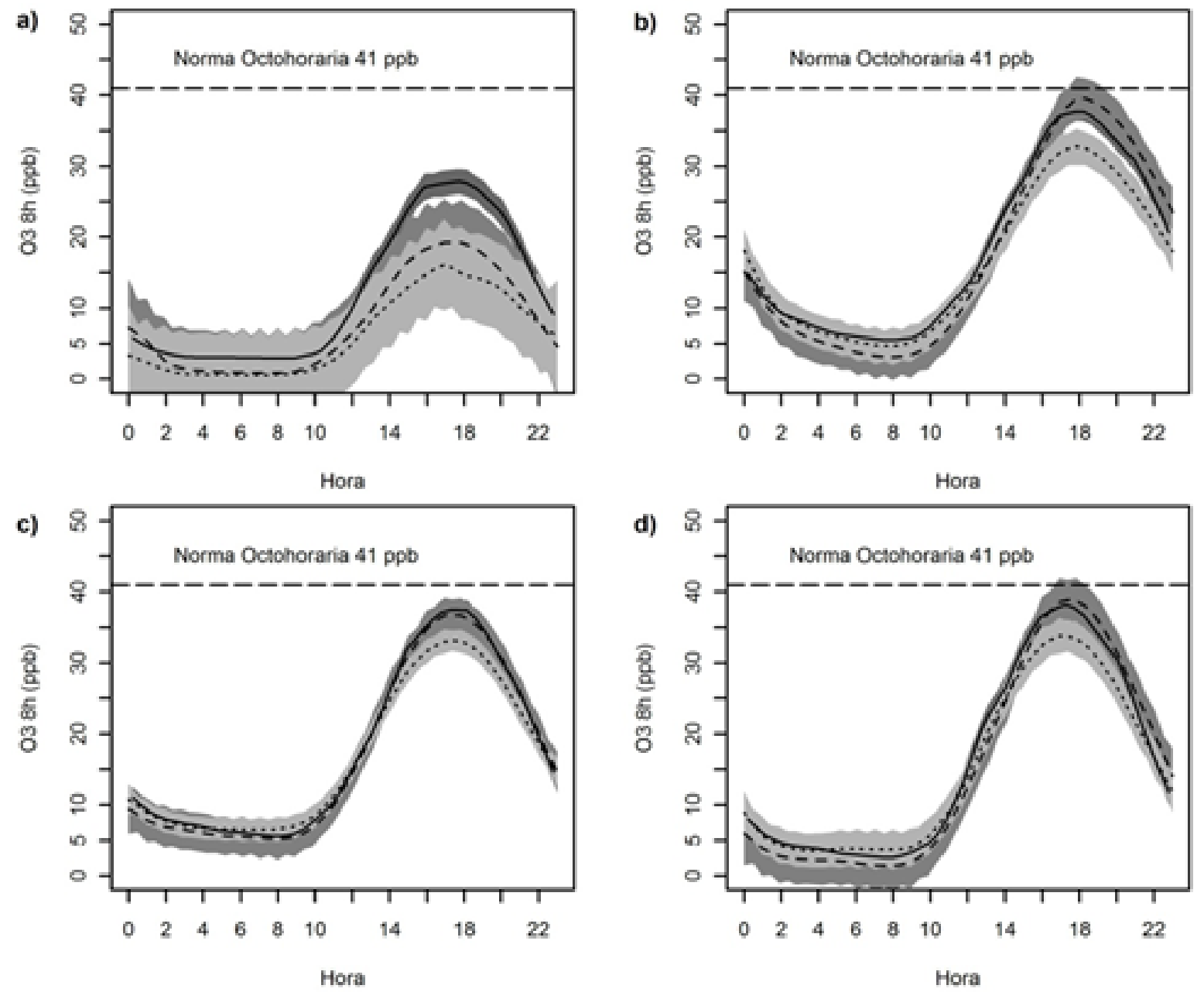

Figura 2. Curvas típicas y bandas de variabilidad de los niveles de concentración octohoraria de O3 en la estación Centro de Diagnóstico Automotor del Valle (a), estación CVC (b), estación Polideportivo el Diamante (c) y estación Univalle (d), para los días Lunes-Viernes (-), Sábados (--) y Domingos-Festivos (...), año 2006.

contaminante sobrepasan los límites máximos de CO 8-h establecidos en la Resolución 601 de 2006.

La Figura 4 ilustra el comportamiento de la contaminación de CO 8-h en cada una de las estaciones a lo largo del año 2006, por tipo de día. Durante el 2006, las concentraciones de CO 8-h no sobrepasaron los límites máximos establecidos en la Resolución 601 de 2006. Al igual que en el año 2003, la zona centro (Calle 15) fue la zona más afectada por este tipo de contaminación en los días Martes-Viernes. La contaminación 8-h generada en los días Lunes en la zona centro, presenta un igual comportamiento que en los días Martes-Viernes, sólo que la contaminación es mucho menor en las primeras horas del día lunes.

\section{Conclusiones}

El comportamiento de la contaminación promedio octohoraria de $\mathrm{O}_{3}$ se refleja en una curva con forma sinusoidal en todos los tipos de días, con un pico máximo de concentración que ocurre a las 5 de la tarde. En las horas en que se incrementa la concentración 8-h de $\mathrm{O}_{3}$ troposférico también aumenta la variabilidad de la misma.

A pesar de que la estación CDAV se encuentra cercana a la zona industrial de Acopi Yumbo, esta estación solo presentó las mayores concentraciones 8-h de $\mathrm{O}_{3}$ en durante el 2003, ya que en el 2006, las mayores concentraciones 8-h de $\mathrm{O}_{3}$ se presentaron en la estación que se encuentra a las afueras de la ciudad, con valores 


\section{a)}

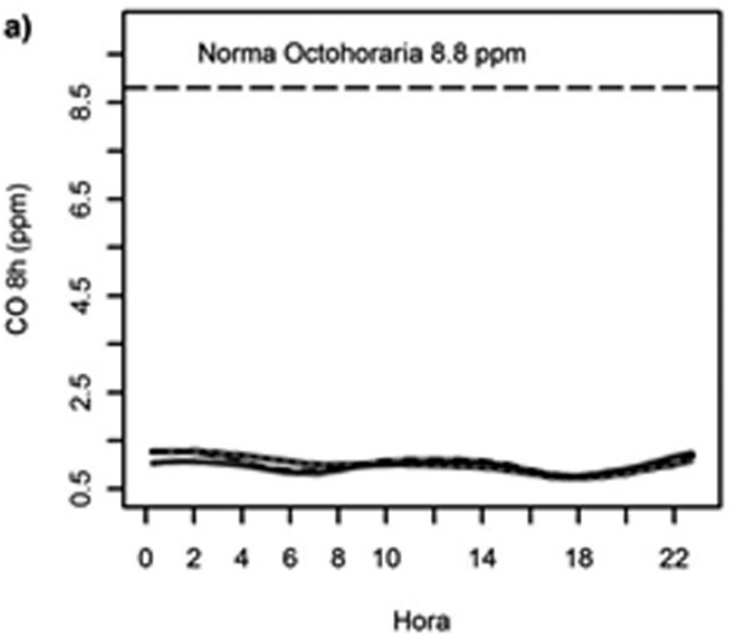

c)

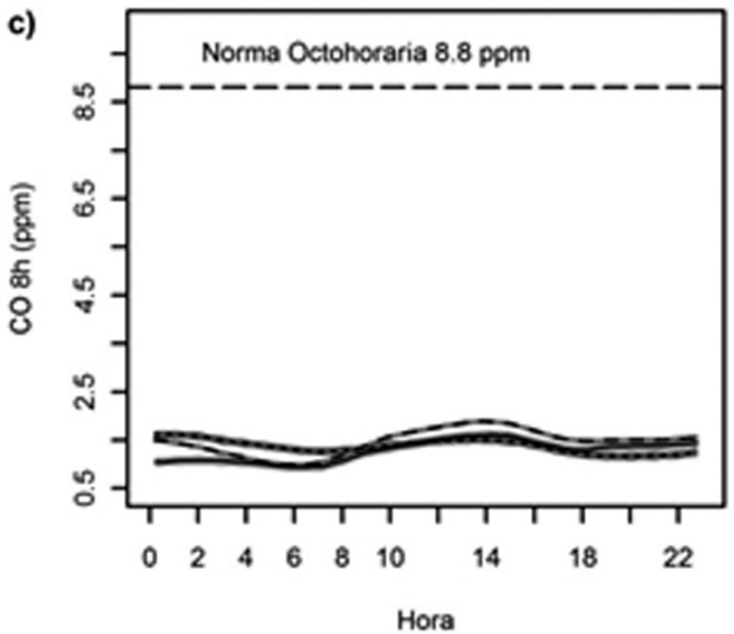

b)

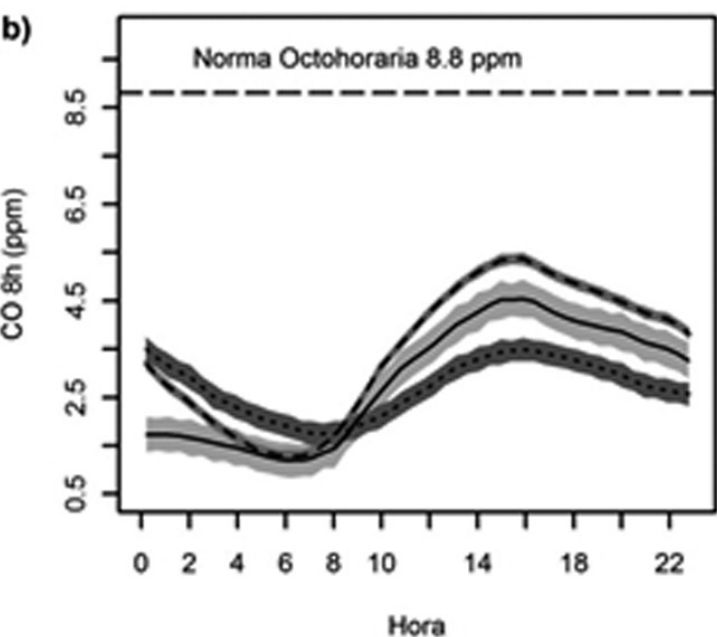

d)

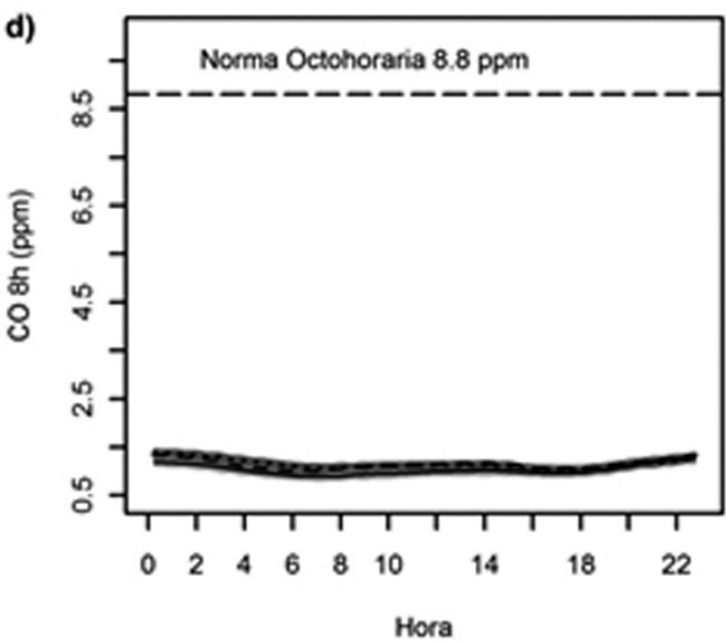

Figura 3. Curvas típicas y bandas de variabilidad de los niveles de concentración octohoraria de CO en la estación Base Aérea (a), estación Calle 15 (b), estación Escuela República de Argentina (c) y estación Polideportivo el Diamante (d), para los dias Lunes (-), Martes-Viernes (--) y días Fines de Semana (...), año 2003.

que sobrepasan el nivel máximo permitido por la Resolución 601 de 2006.

En ninguna de las estaciones de vigilancia de la ciudad de Cali, los niveles de contaminación de CO 8-h superaron el límite máximo permitido en la Resolución 601 de 2006. Los niveles de concentración de $\mathrm{CO}$ 8-h en los fines de semana fueron más bajos que en los días lunes y martes-viernes a partir de las 9:00 a.m.

Esta observación contribuye a reforzar la hipótesis de que la fuente principal de contaminación 8-h de $\mathrm{CO}$ en Cali son las fuentes móviles, debido a que se produce una disminución ostensible de estas fuentes durante los fines de semana. Futuros estudios de este tipo podrían incluir las posibles incidencia de otras fuentes, por ejemplo las planta industriales.

Las bandas de variabilidad muestran, en casi todos los casos, que la contaminación de $\mathrm{CO}$ 8-h presenta un comportamiento diferente dependiendo del tipo de día y zona de vigilancia, por lo que los días fines de semana presentaron los niveles de CO 8-h más bajos y la zona centro de la ciudad de Cali reflejó la mayor contaminación de $\mathrm{CO}$ 8-h.

En los Sistemas de Vigilancia de la Calidad del Aire existe la posibilidad de que se presente el fenómeno de dependencia espacial entre estaciones de vigilancia, por lo que trabajos futuros podrían incorporar elementos de modelación conjunta. 
a)

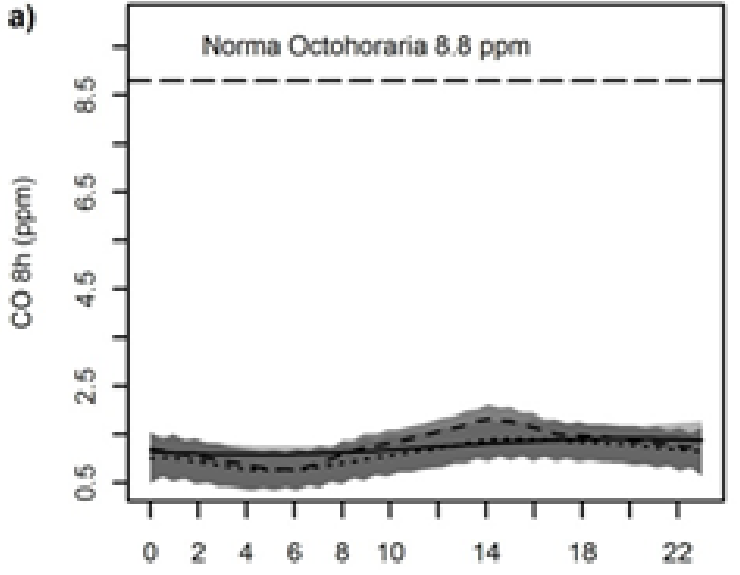

Hora

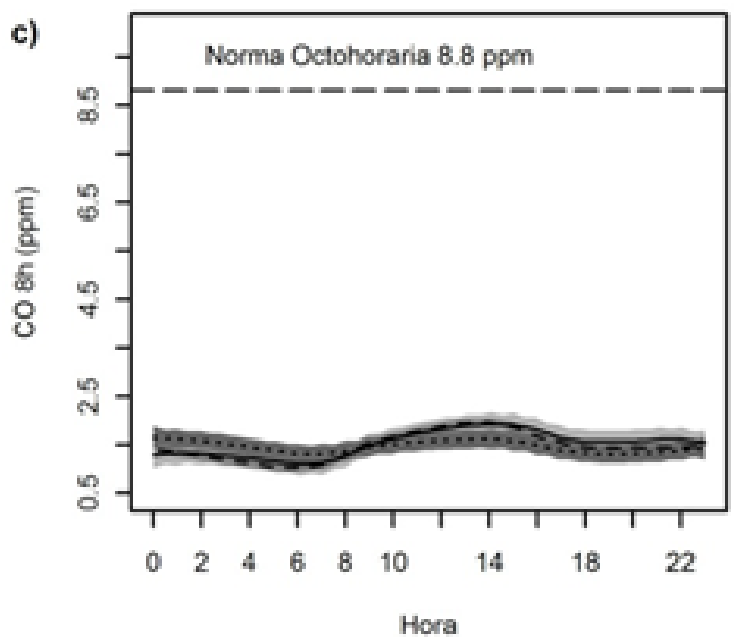

b)
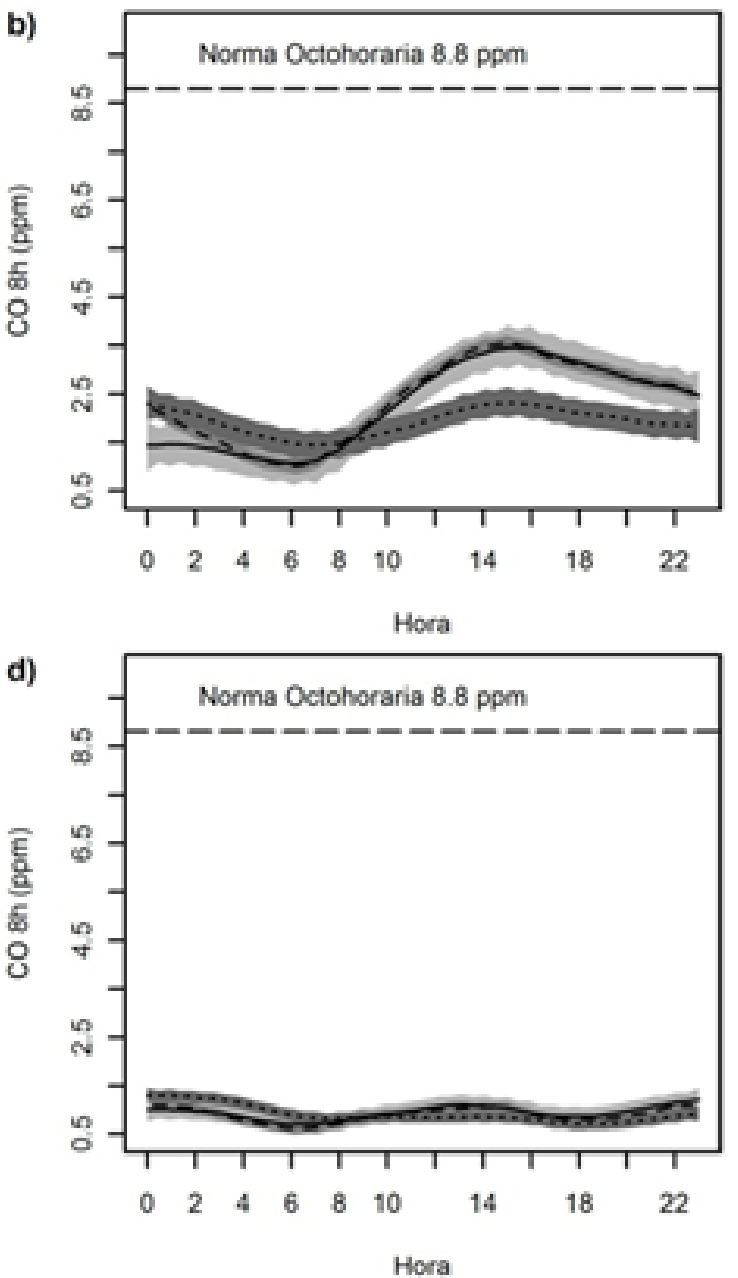

Figura 4. Curvas típicas y bandas de variabilidad de los niveles de concentración octohoraria de CO en la estación Base Aérea (a), estación Calle 15 (b), estación Escuela República de Argentina (c) y estación Polideportivo el Diamante (d), para los dias Lunes (-), Martes-Viernes (--) y días Fines de Semana (...), año 2006.

\section{Referencias Bibliográficas}

Bowman, A., \& Azzalini, A. (1997). Applied smoothing techniques for data analysis: the kernel approach with S-Plus illustrations. Oxford: Oxford University Press.

Bowman, A. W. and Azzalini, A. (2010). $\mathrm{R}$ package 'sm': nonparametric smoothing methods (version 2.2-4)

http://www.stats.gla.ac.uk/ adrian/sm http://azzalini.stat.unipd.it/Book_sm

Cleveland, W.S. (1979). Robust Locally Weighted Regression and Smoothing Scatterplots. Journal of the American Statistical Association, 74 (368), 829-836.
Eubank, R. (1999). Nonparametric regression and spline smoothing. New York: Marcel Dekker, second edition.

Gasser, Th. \& Müller, H.G. (1979). Kernel estimation of regression functions. Smoothing techniques for curve estimation, Ed. Gasser, Th. and Rosenblatt, M., Lecture Notes in Mathematics 757, 23-68.

Gasser, T., Sroka, L., \& Jennen-Steinmetz, C. (1986). Residual Variance and Residual Pattern in Nonlinear Regression. Biometrika, 73 (3), 625-633.

Hall, P. \& Carroll, J. (1989). Variance Function Estimation in Regression: The Effect of Estimating the Mean. Journal of the Royal 
Statistical Society. Series B (Methodological), 51, (1), 3-14.

Härdle, W. (1992). Applied Nonparametric Regression. Cambridge: Cambridge University Press.

IDEAM (Instituto de Hidrología, Meteorología y Estudios Ambientales). (2007). Informe Anual sobre el Estado del Medio Ambientey los Recursos Naturales Renovables en Colombia: Calidad del Aire. Instituto de Hidrología, Meteorología y Estudios Ambientales, Bogotá, D.C.

Nadaraya, E. A. (1964). On estimating regression. Theory of Probability \& Its Applications, 9(1), 141-142.

Olaya, J. (2005). Suavización y regresión no paramétrica. XV Simposio Nacional De Estadística, Paipa, Colombia, p.107 - 129.

Pantazopoulou, A., Katsouyanni, K., KoureaKremastinou \& J., Trichopoulos, D. (1995). Short-term effects of air pollution on hospital emergency outpatient visits and admissions in the greater Athens, Greece area. Environmental Research, 69 (1), 31-36.

Poloniecki, J. D., Atkinson, R. W., Ponce de Leon \& A., Anderson, H. R. (1997). Daily time series for cardiovascular hospital admissions and previous day's air pollution in London, UK. Occupational and Environmental Medicine, 54 (8), 535-540.

Priestley, M. B. \& Chao, M. T. (1972). NonParametric function fitting. Journal of the Royal Statistical Society. Series B (Methodological), 34, 385-392.

R Core Team (2012). R: A language and environment for statistical computing. R Foundation for Statistical Computing, Vienna,
Austria. ISBN 3-900051-07-0, URL http:// www.R-project.org/.

Schwartz, J. (1999). Air pollution and hospital admissions for heart disease in eight U.S. counties. Epidemiology, 10, 17-22.

Seifert, B. \& Gasser, T. (1993). Nonparametric Estimation of Residual Variance Revisited, Biometrika, 80 (2), 373-383.

U.S. CPSC (U.S. Consumer Product Safety Commission). (2013). Horario de verano = Reemplazar las baterías de las alarmas detectoras de humo y de monóxido de carbono. Washington, D.C.

http://www.cpsc.gov/es/Newsroom/ News-Releases/2013/Horario-deverano--Reemplazar-las-baterias-de-lasalarmas-detectoras-de-humo-y-de-monoxidode-carbono/

U.S. EPA (U.S. Environmental Protection Agency). (1990). Clean Air Act Amendments of 1990.

U.S. EPA (U.S. Environmental Protection Agency). (2000). Air Quality Criteria for Carbon Monoxide. U.S. Environmental Protection Agency, Research Triangle Park, NC,

U.S. EPA (U.S. Environmental Protection Agency). (2003). Good Up High, Bad Nearly, Washington, DC.

U.S. EPA (U.S. Environmental Protection Agency). (2006). Air Quality Criteria for Ozone and Related Photochemical Oxidants. U.S. Environmental Protection Agency, Research Triangle Park, NC.

U.S. Department of Labor Occupational Safety and Health Administration. (2002). Carbon Monoxide Poisoning. 